Since the cosine integral is to be employed for an even number of causes and the sine integral for an odd number, the formula $(a)$ and $\left(\boldsymbol{a}^{\prime}\right)$ are sufficient in any case and should have been employed in place of (23) and (23'). Their second or finite term agrees exactly with the special values of (23) and (23') for integer values of $m$. It follows then that the results given in the text are correct as far as this finite term is concerned. It remains to be examined whether the infinite term has any influence on the complete result. Now we have if $b$ is negative

$$
\begin{aligned}
\int_{0}^{\infty} \frac{\mathrm{d} x}{x^{2 n}} \cos b x=\frac{\mathrm{I}}{(2 n-\mathrm{I}) \Delta x^{2 n-1}}-\frac{\pi b^{2 n-1}}{2(2 n-1) ! \cos n \pi} & (\Delta x=0) \\
+ & \int_{0}^{\infty} \frac{\mathrm{d} x}{x^{2 n+1}} \sin b x=\frac{-(-b)}{(2 n-1) \Delta x^{2 n-1}}-\frac{\pi b^{2 n}}{2(2 n) ! \sin (n+1 / 3) \pi} \quad(\Delta x=0)
\end{aligned}
$$

If then for a special value of $\Delta$ a number of angles: $(\Delta-[ \pm \lambda]) u$ becomes negative only the finite term changes sign and the infinite term remains of the same sign whatever value $\Delta$ may have. If then there are $2 n$ simple causes the sum of all the infinite terms is always

$$
\frac{(1-1)^{2 n}}{(2 n-1) \Delta x^{2 n-1}}=0
$$

and if there are $2 n+\mathbf{I}$ simple causes it is

$$
\frac{\Sigma(b)}{(2 n-1) \Delta x^{2 n-1}}=\frac{\sum[(\Delta-[ \pm \lambda]) \cos (2 n+1-[ \pm \mathrm{I}]) \pi]}{(2 n-1) \Delta n^{2 n-1}}=0
$$

It follows then that if only the finite term of $(a)$ and $\left(a^{\prime}\right)$ are used (as was done in the text) a correct result is obtained although each single term was not correctly integrated.

Chas. H. Kummell.

\title{
Observation de la comète Cruls à Louvain le 2 Octobre.
}

Le 2 Octobre, de $17^{\mathrm{h}}$ à $17^{\mathrm{h}} 30^{\mathrm{m}}$, j'ai observé la comète Cruls par un ciel défavorable. J'ai trouvé la comète à l'aide de bonnes jumelles: le noyau apparaissait comme une étoile de $\mathrm{I}^{\text {ère }}$ grandeur et la queue, quoique très visible, ne se prolongeait qu'à une distance de $I^{\circ}$ environ, à cause des légères vapeurs qui garnissaient l'éclaircée imparfaite dont j'ai pu profiter. Ces vapeurs étaient assez épaisses pour rendre l'astre presque invisible ensuite dans le chercheur de ma lunette de Secretan. Avec le grossissement de 38 fois je ne pouvais voir que le noyau et la chevelure; mais chose singulière, quoique la mise au point fût bonne, le noyau paraissait double; l'ap̀parence d'un noyau supplémentaire se trouvait du côté opposé au soleil et était peut-être due à un éclat plus grand de la queue dans cette région. En tous cas je me suis assuré que, dans la portion du ciel occupée par la comète, aucune étoile assez brillante n'aurait pu produire cette apparence en se trouvant dans le voisinage du noyau.

\begin{tabular}{|c|c|c|c|c|c|}
\hline 1882 & M. Z. Kiel & 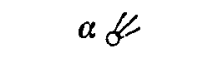 & $\log p .4$ & $\delta \not 4$ & $\log p .4$ \\
\hline $\begin{array}{ll}\text { Oct. } & 5 \\
& 6\end{array}$ & $\begin{array}{l}\text { I } 7^{\mathrm{h}} 10^{\mathrm{m}} 37^{\mathrm{s}} \\
\mathrm{I} 7 \mathrm{I} 35^{\circ}\end{array}$ & $\begin{array}{l}10^{\mathrm{h}} 3^{2^{\mathrm{m}}} 55^{\mathrm{s}} .84 \\
\text { 10 } 3124.22\end{array}$ & $\begin{array}{l}9 \cdot 5 \circ I_{n} \\
9 \cdot 468_{n}\end{array}$ & $\begin{array}{llll}-9^{\circ} & 14^{\prime} & 20^{\prime \prime} \\
-9 & 4 \mathrm{I} & 20.4\end{array}$ & $\begin{array}{l}0.868 \\
0.878\end{array}$ \\
\hline
\end{tabular}

Louvain 1882 Oct. 8.

Dr. F. Terby.

Beobachtungen des Cometen Cruls am Aequatoreal der Kieler Sternwarte.

Als Vergleichstern wurde October $5 \alpha$ Hydrae und October 6 Hydrae benutzt und die Oerter derselben dem Berliner Jahrbuche entnommen. 